This item was submitted to Loughborough's Research Repository by the author.

Items in Figshare are protected by copyright, with all rights reserved, unless otherwise indicated.

\title{
Altering the speed profiles of wheelchair rugby players with game-simulation drill design
}

PLEASE CITE THE PUBLISHED VERSION

http://dx.doi.org/10.1123/ijspp.2016-0700

\section{PUBLISHER}

(c) Human Kinetics Publishing

\section{VERSION}

AM (Accepted Manuscript)

\section{PUBLISHER STATEMENT}

This work is made available according to the conditions of the Creative Commons Attribution-NonCommercialNoDerivatives 4.0 International (CC BY-NC-ND 4.0) licence. Full details of this licence are available at: https://creativecommons.org/licenses/by-nc-nd/4.0/

\section{LICENCE}

CC BY-NC-ND 4.0

\section{REPOSITORY RECORD}

Rhodes, James M., Barry Mason, Thomas A.W. Paulson, and Vicky Goosey-Tolfrey. 2018. "Altering the Speed Profiles of Wheelchair Rugby Players with Game-simulation Drill Design". figshare.

https://hdl.handle.net/2134/24652. 
1 Title: Game-simulation drill design alters the speed profiles of wheelchair rugby players

2 Submission Type: Original investigation

3 Running Head: Speed profiles of wheelchair rugby training

4 Authors:

5 James M Rhodes, Barry S Mason, Thomas A.W Paulson, Victoria L Goosey-Tolfrey ${ }^{1}$

6 Affiliations:

$7 \quad{ }^{1}$ Peter Harrison Centre for Disability Sport, School of Sport, Exercise and Health Sciences,

8 Loughborough University, UK.

9 Corresponding Author:

10 Barry S Mason

11 Peter Harrison Centre for Disability Sport,

12 School of Sport, Exercise and Health Sciences,

13 Loughborough University, UK.

14 Tel: +44 (0)1509 226387.

15 Email: B.Mason@lboro.ac.uk

16

17 Abstract word count: 246

18 Word count: 3431

19 Number of tables and figures: 3 Tables; 2 Figures

20 


\section{ABSTRACT}

22 Purpose: This study examined the speed profiles of elite wheelchair rugby (WCR) players 23 during game-simulation training drills of differing player number and shot-clock regulations. 24 A secondary aim was to determine whether the profiles were further influenced by players classification. Methods: Eight elite WCR players (low-point $n=3$; high-point $n=5$ ) were monitored using a radio-frequency based, indoor tracking system during training sessions over a 5-month period. Speed profiles were collected for three modified game-simulation drills: i) 3 vs. 3 drills ( $n=8$ observations); ii) 30-s shot-clock ( $n=24$ observations) and iii) 15-s shot-clock ( $n=16$ observations) and were compared with regular game-simulation drills (4 vs. 4, 40-s shot-clock; $n=16$ observations). Measures included mean and peak speed, exercise intensity ratios - defined as the ratio of time spent performing at high and low speeds (H:L) and the number of high speed activities performed. Results: Compared to regular game-simulation drills, 3 vs. 3 drills elicited a moderate increase in mean speed (6.3\%; effect size $[E S]=0.7)$ and the number of high speed activities performed $(44.1 \%$; ES = 1.1). Minimal changes in speed profiles were observed during the 30-s shot clock, although moderate to large increases in all measures were observed during the 15-s shot-clock drills. Classification-specific differences were further identified, with increased activity observed for high-point players during the 3 vs. 3 drill and for low-point players during the 15-s shotclock. Conclusion: By reducing the number of players on court and the shot-clock to 15-s coaches can significantly increase the speed profiles of elite WCR players during gamesimulation drills.

Keywords: Physical preparation, classification, Paralympic, training, elite athletes 
Wheelchair rugby (WCR) is a Paralympic team sport played by individuals with an impairment that affects both upper and lower limbs such as spinal cord injuries, multiple amputations, cerebral palsy and neuro muscular diseases. ${ }^{1}$ Given the diversity of impairments, players are classified using a point score depending on the severity of their impairment ranging from 0.5 (most impaired) to 3.5 (least impaired). Teams are made up four players whose total point score must not exceed 8 points at any given time. WCR is played over 8minute quarters using a 'game-clock' whereby the time is stopped whenever a goal is scored or an offence is committed. Other time regulations exist through the use of a shot-clock, whereby teams have 40 -seconds to score a goal once the ball has been inbounded and must advance past the half-way line within 12-seconds otherwise possession is conceded. ${ }^{2}$

Recent research has revealed that WCR is an intermittent sport with players typically covering distances of 2500-4600 m during competition, ${ }^{3,4}$ with the majority of time spent performing low speed activities interspersed with frequent bouts of high speed activities. ${ }^{3}$ Classification has also been shown to affect activity profiles during competition with greater distances covered and higher peak speeds reached in higher classification players. Moreover, low-point players $(\leq 1.5)$ spend more time at very low speeds, yet perform more frequent high speed activities than high-point players $(\geq 2.0) .{ }^{3}$ Furthermore, the ability to reach high peak speeds and perform a greater number of high speed activities have been associated with successful performance, specifically in the high-point players. ${ }^{5}$ Subsequently, the physical demands of WCR competition have been relatively well documented. The physical preparation of athletes requires a multi-disciplinary approach to session planning whereby coaches and practitioners manipulate the training environment to replicate the demands of international competition. ${ }^{6}$ However very few studies have explored the physical demands of WCR training. ${ }^{7-9}$ Game-simulation drills are a popular training modality with coaches from a 
range of team sports because they enable a combination of technical, tactical and physical elements of performance to be developed under competition-specific conditions and were shown to account for $44 \%$ of total training time in WCR. ${ }^{9}$ Rhodes et al. ${ }^{9}$ also revealed that game-simulation drills offered the closest representation of the speed profiles observed during elite competition compared to conditioning (continuous pushing drills designed to develop physical capacity), game-related (half-court drills with coach interaction designed to develop tactical plays under game-specific conditions) and skill-based drills (structured drills designed to develop ball-handling skills). Despite this, subtle differences in speed profiles were observed between game-simulation drills depending on the duration and timing method enforced. It was revealed that 3- and 8-minute game-simulation drills using a 'game-clock' underrepresented the peak speeds and high speed activities observed during competition. ${ }^{9}$ Alternatively a 10-minute running clock, whereby timing is continuous and not paused at any point, provided a better representation of competition-specific speed profiles, irrespective of classification. ${ }^{9}$ While such results have provided an insight into current WCR training practice, it is currently unclear how game-simulation drills can best be utilised to prepare elite WCR players.

Research in able-bodied team sports have extended the examination of gamesimulation drills to explore the impact of manipulating the number of players on court during game-simulation drills ${ }^{10-16}$ and modified game rules ${ }^{12}$ on players' activity profiles. By reducing player numbers, players have been shown to spend more time performing high speed activities during rugby- ${ }^{14}$ and soccer-specific ${ }^{15}$ drills. Unfortunately no such information exists with regards to WCR. It is also essential to understand the variability typically observed in activity profiles to determine whether any differences between training modalities are meaningful, which has been adopted by research into able-bodied team sports. ${ }^{17-19}$ Subsequently the aim of the current study was to firstly determine the between 
observation variability in speed profiles during regular WCR game-simulation drills. The primary objective was then to compare the speed profiles of elite WCR players during gamesimulation drills, whilst modifying both player number and shot-clock regulations, in relation to regular game-simulation drills. A secondary aim was to determine whether these comparisons were further affected by classification. This information can support coaches and/or sports science practitioners in the periodisation of court-based training in preparation for competition.

\section{METHODS}

\section{Participants}

105 Eight international WCR players (age: $27.3 \pm 5.3$ years; mass: $60.9 \pm 10.2 \mathrm{~kg}$ ) provided 106 written informed consent and volunteered to participate in this study. Players were grouped 107 by their International Wheelchair Rugby Federation (IWRF) classification as either low-point 108 ( $\leq 1.5$; LP: $n=3$ ) or high-point players ( $\geq 2.0$; HP; $n=5$ ). Written informed consent was 109 obtained from all players and approval for the study was obtained by the University's ethical 110 advisory committee.

\section{Procedures}

112 Data were collected during an elite international WCR squad's training sessions over a 5113 month period throughout the competitive phase of the season (January to May; 30 training 114 observations). In consultation with the Head Coach and Sport Scientist, three separate 115 variations of WCR-specific game-simulation drills were developed and the subsequent speed 116 profiles were compared to regular game-simulation drills. Speed profiles were monitored for 117 all player's on-court using a radio-frequency based Indoor Tracking System [ITS] (Ubisense, 118 Cambridge, UK) as previously described and validated for use within WCR. ${ }^{20}$ Players were 
119 equipped with a small, lightweight tag (size $=40 \times 40 \times 10 \mathrm{~mm}$; mass $=25 \mathrm{~g}$ ) sampling at 8

$120 \mathrm{~Hz}$, positioned on or near the foot-strap of each player's rugby wheelchair. Each player wore

121 the same tag unit during all testing sessions to exclude any potential tag variability. Players

122 were instructed to maintain normal fluid intake and cooling strategies, and no additional

123 dietary interventions were undertaken. All players were familiar with the ITS and its

124 operational procedures.

125

Players were allocated into balanced teams according to their physical, technical and

126 tactical proficiency, as determined by the head coach and also according to their IWRF

127 classification for each drill. The maximum classification permitted was reduced from 8.0

128 points to 6.0 points during the 3 vs. 3 format to ensure that teams were balanced according to

129 their classification. The court size (28 m x 15 m), clock format (running-clock), team line-ups

130 and the overall duration (10-minute quarters) of all drills were strictly controlled based on

131 findings from previous research. ${ }^{9}$ Three game-simulation drills were developed, whereby the

132 player number and shot-clock regulations were modified. These included a) 3 vs. 3 game

133 format ( $n=8$ training observations); and modifications to the shot-clock regulations to

134 evaluate b) 30-second shot-clock ( $n=24$ training observations); and c) 15-second shot-clock

135 ( $n=16$ training observations) during 4 vs. 4 game formats. The shot-clock was started once a

136 team were in possession of the ball, of which they had the stipulated time to score otherwise,

137 they conceded possession. The speed profiles of all modified drills were compared to that of 138 regular game-simulation drills with a 10-minute running-clock format and 40-second shot

139 clock ( $n=16$ observations). The order in which the drills were performed were randomly

140 varied across the collection period, with a training observation characterised for each

141 individual as the accumulation of activity observed during the respective four quarters of that

142 drill. Speed profiles for game-simulation drills were therefore presented as the mean of all

143 training observations for each individual player. Game-simulation drills were preceded by a 
144 20-minute standardised warm-up involving moderate- to high-intensity continuous pushing,

145 dynamic stretching and maximal linear sprints. Coaches verbally encouraged the players

146 throughout the drills.

\section{Measures}

148 Mean and peak speed $\left(\mathrm{m} \cdot \mathrm{s}^{-1}\right)$ were analysed during the aforementioned training drills for 149 each player. The relative time spent in five arbitrary speed zones was calculated based upon 150 the percentage of each player's mean peak speed attained during the regular game-simulation 151 drills. The percentage thresholds as previously used in team sports, ${ }^{3,21}$ were: very low ( $\leq$ 152 20\%), low (21-50\%), moderate (51-80\%), high (81-95\%) and very high (> 95\%). These 153 thresholds were subsequently used to calculate the ratio of time spent performing high speed 154 activities (high and very high speed zones) in relation to low speed activities (very low and 155 low speed zones) to determine the exercise-intensity ratio (H:L) as used previously within WCR. ${ }^{9}$ Further analysis of the combined time spent in high and very high speed zones was 157 extended to include the time spent $(\%)$, relative number $\left(\mathrm{n} \cdot \mathrm{min}^{-1}\right)$, and the mean distance $(\mathrm{m})$ 158 and duration (s) of high speed activities.

\section{Statistical Analyses}

160 Data were processed and analysed using a customised Excel spreadsheet (Microsoft,

161 Redmond, USA). Data are presented as mean \pm SD for each parameter reported. Between162 observation variability of the measures was calculated from the regular game-simulation 163 drills (10-minute running-clock, 40-second shot clock) from a larger cohort of athletes $(n=$

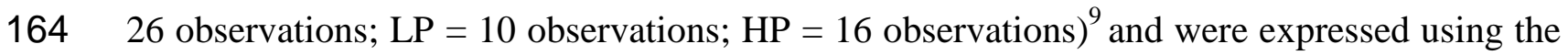
165 coefficient of variation (CV [\%]) and presented with 95\% confidence limits (CL) as markers 166 of the estimates uncertainty. Effect sizes (ES) were calculated as the ratio of the mean 167 difference to the pooled standard deviation of the difference. Data were interpreted using ES 
168 with 95\% confidence intervals $( \pm \mathrm{CI}$ ) and the percentage change to determine the magnitude 169 of effects, classified as trivial $<0.2$; small 0.2 to 0.6 ; moderate 0.6 to 1.2 ; large 1.2 to 2.0 ; and 170 very large $>2.0 .^{22}$ The smallest worthwhile change (SWC; \%) in speed profiles were defined

171 as 0.2 multiplied by the between subject standard deviation. ${ }^{23}$ The SWC in measures were 172 again calculated from the larger cohort, and can be used to interpret the magnitude of effects 173 reported here. This statistical approach was utilised to identify worthwhile changes in 174 performance while accounting for the variability of measurement.

175

\section{RESULTS}

177 The between-observation variability and SWC in speed profiles are reported in Table 1.

178 Overall, variability was greatest for the time spent performing high speed activities (22.4\% $179 \mathrm{CV})$ and the relative number of high speed activities performed (16.0\% CV). Lowest 180 variability was observed for mean (1.9\% CV) and peak speed values (2.4\% CV). Between181 observation variability was also shown to be greater in LP compared to HP (Table 1).

Table 2 illustrates the speed profiles observed during modified game-simulation drills 186 in relation to regular game-simulation drills, with Figure 1 demonstrating the magnitude of any differences between these drills. Compared to regular game-simulation drills, the 3 vs. 3 drills elicited a moderate increase in mean speed (6.3\%; ES $=0.6$; $95 \%$ CI 0.4 to 0.8 ), the exercise-intensity ratio (15.1\%; ES $=0.8$; $95 \%$ CI 0.5 to 1.1 ), time spent performing high speed activities (44.1\%; ES $=1.1 ; 95 \%$ CI 0.8 to 1.4 ) and the relative number of high speed 
191 activities (43.5\%; ES = 1.0; 95\% CI 0.8 to 1.2) performed (Figure 1). Only trivial to small 192 increases in speed profiles were observed during the 30-second shot-clock compared to 193 regular game-simulation drills (Figure 1). Large increases in mean speed (12.9\%; ES = 1.5; $19495 \%$ CI 1.4 to 1.6), peak speed (8.8\%; ES = 1.3; 95\% CI 1.1 to 1.5) and the relative number 195 of high-intensity activities performed (57.1\%; ES = 1.3; 95\% CI 1.0 to 1.6) were observed 196 during the 15-second shot clock in relation regular game-simulation drills. Moderate 197 increases in the exercise-intensity ratio (15.1\%; ES $=0.6$; 95\% CI 0.3 to 0.9 ) and the time spent performing high speed activities (27.1\%; ES $=0.8$; 95\% CI 0.6 to 1.0 ) were also 199 observed during the 15-second shot-clock (Figure 1).

200

201

TABLE 2 ABOUT HERE

202

FIGURE 1 ABOUT HERE

203

204

Table 3 demonstrates the speed profiles of both classification groups during the 205 modified game-simulation drills compared to regular game-simulation drills, with the 206 corresponding effect sizes illustrated in Figure 2. When categorised by classification, the 3 vs. 2073 drills elicited large increases in mean speed (10.1\%; ES = 1.6; 95\% CI 1.4 to 1.8) and the 208 exercise-intensity ratio (18.4\%; ES $=1.2$; 95\% CI 1.0 to 1.4) and a moderate increase in the 209 relative number of high speed activities performed (25.0\%; ES $=1.0$; 95\% CI 0.8 to 1.2$)$ for 210 HP compared with regular game-simulation drills. During the 30-second shot-clock drill, 211 moderate to large decreases in peak speed (1.7\%; ES $=0.7 ; 95 \%$ CI 0.5 to 0.9$)$, the relative 212 time spent performing high speed activities (22.6\%; ES = 1.4; 95\% CI 1.1 to 1.7) and the 213 mean distance (21.0\%; ES = 1.1; 95\% CI 0.9 to 1.3) and duration (22.9\%; ES = 1.1; 95\% CI 
2140.8 to 1.4) of these activities were observed in relation to regular game-simulation drills for 215 LP (Figure 2). Alternatively, the 15-second shot-clock drills elicited large to very large 216 increases in mean speed (19.7\%; ES = 2.0; 95\% CI 1.8 to 2.2), peak speed (11.9\%; ES = 1.8; $21795 \%$ CI 1.6 to 2.0), exercise-intensity ratio (40.9\%; ES = 2.3; 95\% CI 2.0 to 2.6), the time 218 spent performing high speed activities (41.9\%; ES $=1.4$; 95\% CI 1.1 to 1.7 ) and the relative 219 number of high-intensity activities (62.5\%; ES = 1.4; 95\% CI 1.2 to 1.6) performed by LP 220 (Figure 2). For HP, large increases in mean speed (10.0\%; ES = 1.2; 95\% CI 1.0 to 1.4), peak speed (7.4\%; ES = 1.3; 95\% CI 1.1 to 1.5$)$ and the relative number of high-intensity activities 222 performed (50.0\%; ES = 1.2; 95\% CI 1.0 to 1.4) were observed in relation to regular game223 simulation drills.

TABLE 3 ABOUT HERE

FIGURE 2 ABOUT HERE

\section{DISCUSSION}

229 Changes in player number and shot-clock regulations both influenced the speed profiles of 230 elite WCR players during game-simulation training drills. Classification-specific differences in speed profiles were also identified between drills, most notably during the 3 vs. 3 and 15second shot clock manipulations for LP and HP respectively. These findings provide an evidence-base upon which to plan and periodise classification-specific training strategies to prepare players for international competition. 
and peak speed $(2.4 \% \mathrm{CV})$ were shown to be the most stable measures of speed profiles during WCR game-simulation drills, which were similar to the variations reported in peak speed for soccer $(2.4 \% \mathrm{CV}),{ }^{17}$ rugby league $(3.6 \% \mathrm{CV})^{18}$ and Australian football $(5.3 \%$

$240 \mathrm{CV}) .{ }^{19}$ In contrast, the variability in high speed activities observed in the current study (16$24139 \%$ CV) was slightly greater compared to the aforementioned studies (18-20\% CV). Such 242 findings are likely attributable to the notable differences in impairment severity, positional243 roles ${ }^{3,5,24}$ and physical capacity ${ }^{25}$ between players that may influence this variability in high 244 speed profiles. Despite the small cohort of players, descriptive data in relating to 245 classification revealed clear differences in the responses of LP and HP to different drill 246 manipulations. It was originally considered that greater variations in speed profiles would be 247 experienced by HP since they are typically more involved in the play than LP. ${ }^{5,24}$ However 248 this was not the case as the largest variations were in fact observed for LP. The speed profiles 249 of LP are thought to be influenced as much by their defensive role on court as opposed to 250 their functional capabilities ${ }^{5}$ and as such their roles may have been influenced even further by 251 the modifications to player number and shot-clock regulations thus accounting for the larger 252 between-observation variability. Regardless of classification, these findings suggest caution 253 is required when using high speed activities to interpret changes in a WCR players 254 performance.

Reducing the number of players on court from 4 vs. 4 to 3 vs. 3 whilst maintaining a 40-second shot clock had a substantial effect on the speed profiles observed during game257 simulation drills. The relative number of high speed activities performed during the 3 vs. 3 258 drills increased with a moderate effect compared to regular game-simulation drills. Even 259 though this measure demonstrated large variability $(16.0 \% \mathrm{CV})$, the magnitude of change 260 detected was twice the CV\%, and several orders of magnitude larger than the SWC for this parameter. The greater available court-ratio per player during the 3 vs. 3 drills $\left(70.0 \mathrm{~m}^{2}\right.$ vs. 
$26252.5 \mathrm{~m}^{2}$ ) was likely to account for the increased speed profiles based on the positive 263 correlations between increased relative pitch-ratio per player and activity profiles in able264 bodied team sports. ${ }^{11,14,16}$ Differences in speed profiles during the 3 vs. 3 drill were further 265 influenced by functional classification. Whilst speed profiles were comparable between 3 vs. 2663 and regular 4 vs. 4 game-simulation drills for LP, HP performed at a higher mean speed and 267 exercise-intensity ratio during the 3 vs. 3 manipulation. The increased activity for HP was 268 likely due to the positional requirements during these drills. As offensive players, HP are 269 responsible for handling the ball and are typically more involved in the play than LP whose 270 defensive role primarily requires them to block opponents. ${ }^{5,24}$ Subsequently, the reduced 271 passing options during 3 vs. 3 drills may instigate an increased activity from HP in order to 272 continually create space to receive the ball. These findings suggest that 3 vs. 3 drills provide a 273 greater stimulus for developing both aerobic and anaerobic capabilities in training than 274 regular game-simulation drills, especially in HP. 276 profiles of WCR players. Compared to regular game-simulation drills, the 30-second shot277 clock increased the mean speed of players by $~ 3 \%$. However such a change may have a 278 relatively minor impact on training adaptation as even though minimal variability was 279 reported for mean speed $(1.9 \% \mathrm{CV})$, the magnitude of change detected during these drills was not large enough to interpret as a worthwhile change. Accordingly, it is important that 281 coaches and practitioners interpret changes based on the magnitude of change, rather than a 282 statistical difference. The comparable profiles between the 30-second shot clock and regular 283 game-simulation drills could be due to the fact that the average time of each offensive-play 284 during competition is typically less than 30-seconds ( 23 seconds; unpublished data). 285 Therefore, a reduction from 40- to 30-seconds may not have been substantial enough to 286 significantly alter the activities of WCR players. The comparable speed profiles elicited 
287 during the 30-second shot-clock manipulation drill were not conclusive for LP who 288 experienced a $22.6 \%$ decrease in the time spent performing high speed activities. While such 289 a change was larger than the SWC, the magnitude of change detected here was only 290 marginally greater than the CV for this parameter. Therefore, it is unclear whether this 291 change was a true reflection of the demands of this specific drill.

The greatest change in speed profiles were observed when the shot-clock was further 293 reduced to 15-seconds. Large to very large increases in mean and peak speed values occurred 294 during this manipulation. Furthermore, players performed $~ 57 \%$ more high speed activities 295 during the 15-second shot-clock drills (1.1 per minute) compared to regular game-simulation 296 drills ( 0.7 per minute). The magnitude of change detected during the 15 -second shot-clock drill was again more than double the between-observation variability. However, given the 298 high variability of the number of high speed activities observed in the current study, 299 individual responses should be monitored to ensure that all players receive the intended 300 training stimulus. ${ }^{14} \mathrm{LP}$ actually performed at a greater mean speed $\left(1.43 \mathrm{~m} \cdot \mathrm{s}^{-1}\right)$ and exercise301 intensity ratio (1:2.2) to values previously observed during WCR-specific conditioning drills 302 $\left(1.32 \mathrm{~m} \cdot \mathrm{s}^{-1} ; 1: 2.4\right) .{ }^{9}$ Coaches may therefore achieve the required dose of conditioning for LP, 303 whilst maintaining the sport specificity during the 15-second shot-clock game-simulation drill. 304 An advantage of game-simulation drills is the potential multifunctional training benefit they 305 provide by simultaneously addressing physical, tactical and technical aspects of performance 306 altogether. ${ }^{13}$ However, increasing the speed profiles of drills may also elicit changes in the 307 quality of technical actions. ${ }^{15}$ It is plausible to suggest players may not be able to consistently 308 sustain the technical skills required and as such, training may become counterproductive in 309 terms of technical performance. Unfortunately, this has not been empirically examined and 310 was outside the scope of this study, although worthy of future investigation. 
312 be used to manipulate players' activity profiles during game-simulation drills. For instance,

313 time stipulations could be implemented for teams to cross the half court, to further increase 314 workload. In addition, research into the effects of different number of repetitions of each drill 315 with different rest periods would further benefit coaches with training prescription. As 316 previously reported by Paulson et al. ${ }^{8}$ high speed profiles do not always equate to high 317 physiological loads, since maintaining momentum is physically less demanding than 318 activities requiring high intensity accelerations. Unfortunately the indoor tracking system 319 does not sample at a frequency capable of accurately quantifying acceleration to help 320 investigate this statement. However, the inclusion of mean distance and duration of high 321 speed activities does provide coaches with some insight as to whether changes in speed 322 profiles result from longer or more frequent efforts. Subsequently future research would be 323 advised to collect data about acceleration performance during different drill types, but should 324 also explore the effect that these drills can have on a players internal responses as well as the 325 impact the drills may have on aspects of their technical performance. This would provide 326 coaches with even more detailed information about which drills should be prescribed at 327 specific points throughout the season.

\section{PRACTICIAL APPLICATIONS}

330 Although specific training objectives alter throughout the season, the ultimate objective of

331 training during the competitive phase of the season should be to induce similar responses to 332 those encountered during competition. The current data provide evidence that subtle changes 333 to the design of game-simulation drills can influence the external training responses of elite 334 WCR. Coaches can subsequently impose a greater external load on players simply by 
335 reducing the shot-clock to 15 -sec. A reduction in player numbers from 4 vs. 4 to 3 vs. 3 is 336 another strategy that can be employed by coaches to overload players, although as previously 337 highlighted this effect was more pronounced for HP. Therefore a players classification must 338 also be considered.

339

\section{CONCLUSIONS}

341 The current study demonstrated that reducing the shot-clock from 40- to 30-sec during game342 simulation drills has little bearing on the speed profiles of elite WCR players, whereas a 15343 sec shot clock increased the mean speed and high speed activities experienced by players. A 344 reduction in player numbers from 4 vs. 4 to 3 vs. 3 has a similar impact on the speed profiles 345 of WCR players, although this response was only observed for HP. 
1. International Wheelchair Rugby Federation (IWRF). International wheelchair rugby classification manual $3^{\text {rd }}$ edition revised 2015. Retrieved from http://www.iwrf.com/classification.htm.

2. Orr K, Malone LA. Wheelchair rugby. In: Goosey-Tolfrey, ed. Wheelchair Sport. Leeds, UK: Human Kinetics; 2010:151-166.

3. Rhodes JM, Mason BS, Perrat B, Smith M, Malone LA, Goosey-Tolfrey VL. Activity profiles of elite wheelchair rugby players during competition. Int J Sports Physiol Perform. 2015;10:318-324.

4. Sarro KJ, Misuta MS, Burkett B, Malone LA, Barros RML. Tracking of wheelchair rugby players in the 2008 demolition derby final. J Sports Sci. 2010;28:193-200.

5. Rhodes JM, Mason BS, Malone LA, Goosey-Tolfrey VL. Effect of team rank and player classification on activity profiles of elite wheelchair rugby players. J Sports Sci. 2015;30:2070-2078.

6. White AD, MacFarlane NG. Analysis of international competition and training in men's field hockey by global positioning system and inertial sensor technology. $J$ Strength Cond Res. 2015;29:137-143.

7. Barfield J, Malone L, Arbo C, Jung A. Exercise intensity during wheelchair rugby training. J Sports Sci. 2010;28:389-398.

8. Paulson TAW, Mason B, Rhodes J, Goosey-Tolfrey VL. Individualized internal and external training load relationships in elite wheelchair rugby players. Front Physiol. 2015;6(388):1-7.

9. Rhodes JM, Mason BS, Paulson TAW, Goosey-Tolfrey VL. A comparison of speed profiles between training and competition in elite wheelchair rugby players. Int $J$ Sports Physiol Perform. 2016; In Press.

10. Rampinini E, Impellizzeri FM, Castagna C, Abt G, Chamari K, Sassi A, Marcora SM. Factors influencing physiological responses to small-sided soccer games. J Sports Sci. 2007;25(6):659-666.

11. Castellano J, Casamichana D. Time-motion, heart rate, perceptual and motor behaviour demands in small-sides soccer games: Effects of pitch size. J Sports Sci. 2010;28(14):1615-1623.

12. Hill-Haas SV, Coutts AJ, Dawson BT, Rowsell GJ. Time-motion characteristics and physiological responses of small-sided games in elite youth players: the influence of player number and rule changes. J Strength Cond Res. 2010;24(8):2149-2156.

13. Dellal A, Owen A, Wong DP, Krustrup P, Van Exsel M, Mallo J. Technical and physical demands of small vs. large sided games in relation to playing position in elite soccer. Hum Mov Sci. 2012;31(4):957-969.

14. Kennett DC, Kempton T, Coutts AJ. Factors affecting exercise intensity in rugbyspecific small-sided games. J Strength Cond Res. 2012;26(8):2037-2042.

15. Morgans R, Orme P, Anderson L, Drust B. Principles and practices of training for soccer. J Sport Health Sci, 3(4), 251-257, 2014.

16. Owen AL, Wong DP, Paul D, Dellal A. Physical and technical comparisons between various-sided games within professional soccer. Int J Sport Med. 2014;35(4):286-92.

17. Rampinini E, Coutts AJ, Castagna C, Sassi R, Impellizzeri FM. Variation in top level soccer match performance. Int J Sports Med. 2007;28:1018-1024.

18. Kempton T, Sirotic AC, Coutts AJ. Between match variation in professional rugby league competition. J Sci Med Sport. 2014;17(4):404-407. 
19. Kempton T, Sullivan C, Bilsborough JC, Cordy J, Coutts A J. Match-to-match variation in physical activity and technical skill measures in professional Australian Football. J Sci Med Sport. 2015;18(1):109-113.

20. Rhodes JM, Mason BS, Perrat B, Smith M, Goosey-Tolfrey VL. The validity and reliability of a novel indoor player tracking system for use within wheelchair court sports. J Sports Sci. 2014;32(17):1639-1647.

21. Cahill N, Lamb K, Worsfold P, Headey R, Murray S. The movement characteristics of English Premiership rugby union players. J Sports Sci. 2013;31:229-237.

22. Batterham A, Hopkins W. Making meaningful inferences about magnitudes. Int $J$ Sport Physiol Perform. 2006;1:50-57.

23. Hopkins WG. How to interpret changes in an athletic performance test. Sportscience. 2004;8:1-7.

24. Morgulec-Adamowicz N, Kosmol A, Bogdan M, Molik B, Rutkowska I, Bednarczuk G. Game efficiency of wheelchair rugby athletes at the 2008 Paralympic Games with regard to player classification. Hum Mov. 2010;11:29-36.

25. Morgulec-Adamowicz N, Kosmol A, Molik B, Yilla AB, Laskin J.J. Aerobic, anaerobic, and skill performance with regard to classification in wheelchair rugby athletes. Res Q for Exerc Sport. 2011;82(1):61-69. 


\section{Figure Legends}

420 Figure 1 - Magnitude of change as determined by effect sizes (ES) in (a) speed profiles; and

421 (b) high speed activities during modified game-simulation drills in relation to regular game422 simulation drills. Error bars represent 95\% CI.

423

424 Figure 2 - Magnitude of change as determined by effect sizes (ES) in (a) speed profiles; and 425 (b) high speed activities for LP (black) and HP (grey) during modified game-simulation drills 426 in relation to regular game-simulation drills. Error bars represent 95\% CI. 
a)

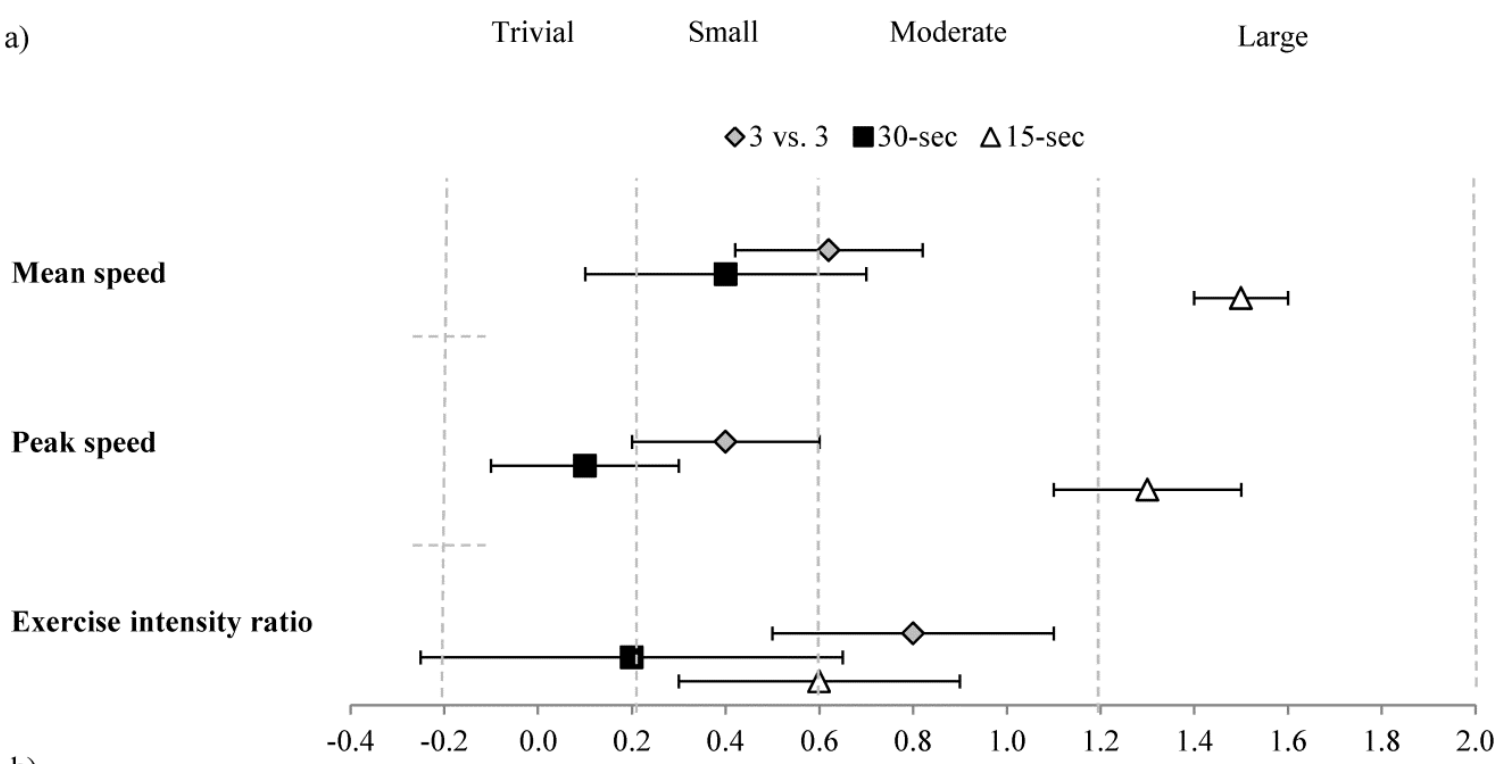

b)

Time spent

Mean distance

Mean duration

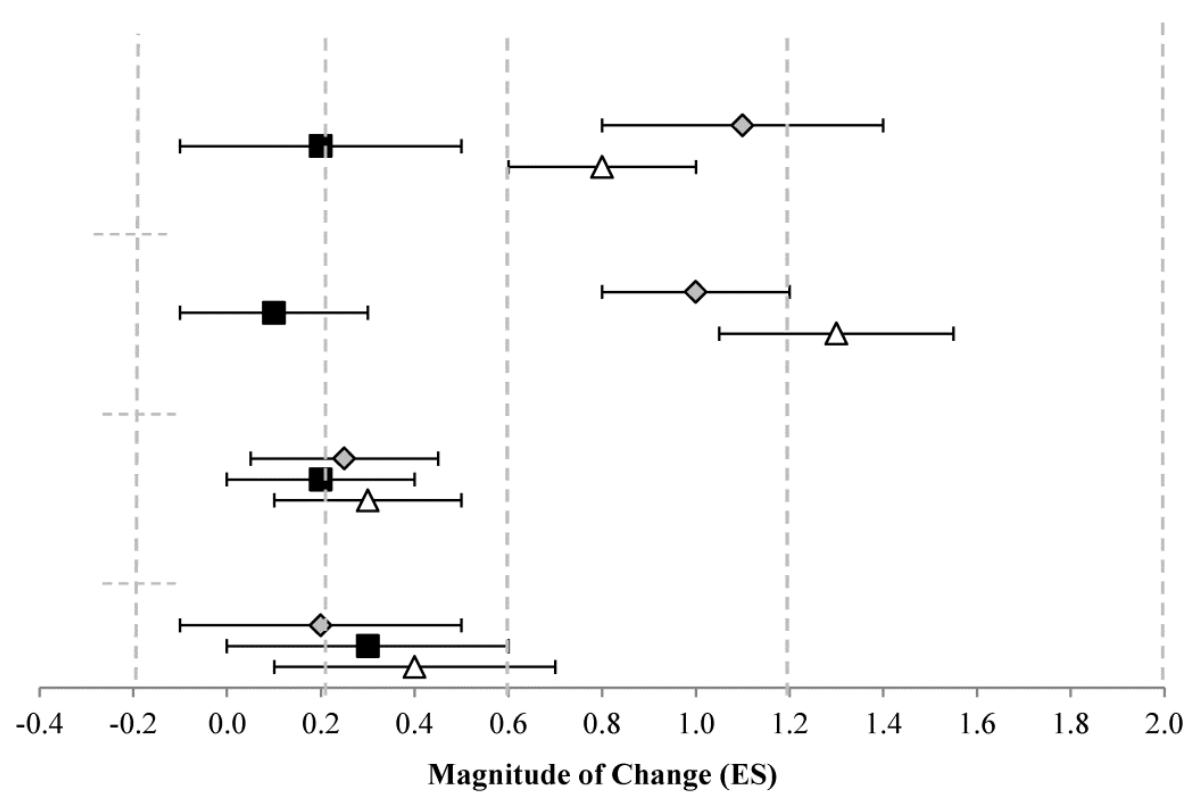

\section{Relative number}

429 
a)

$$
\text { Trivial Small Moderate Large } \quad \text { Very Large }
$$

Mean speed

$\diamond 3$ vs. $3 \quad \square 30$-sec $\Delta 15$-sec

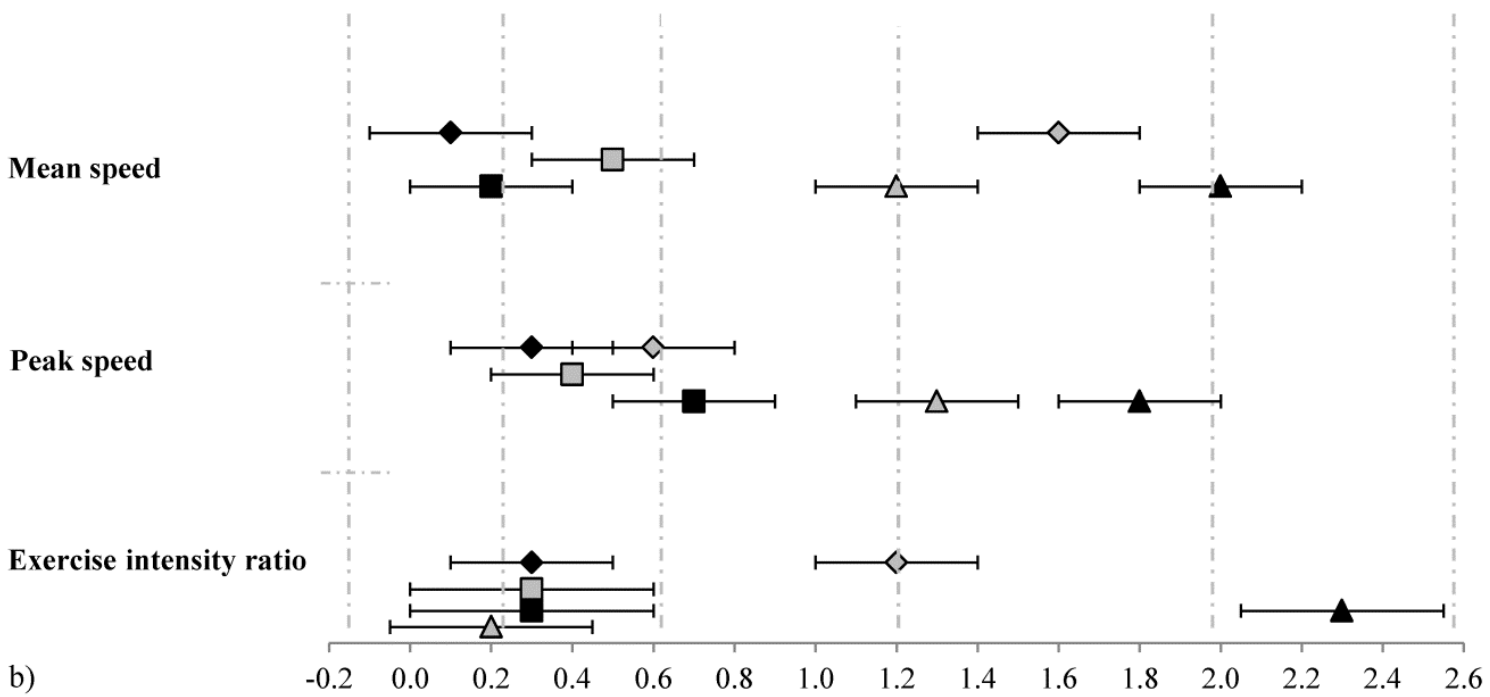

Time spent

Relative number

Mean distance

Mean duration

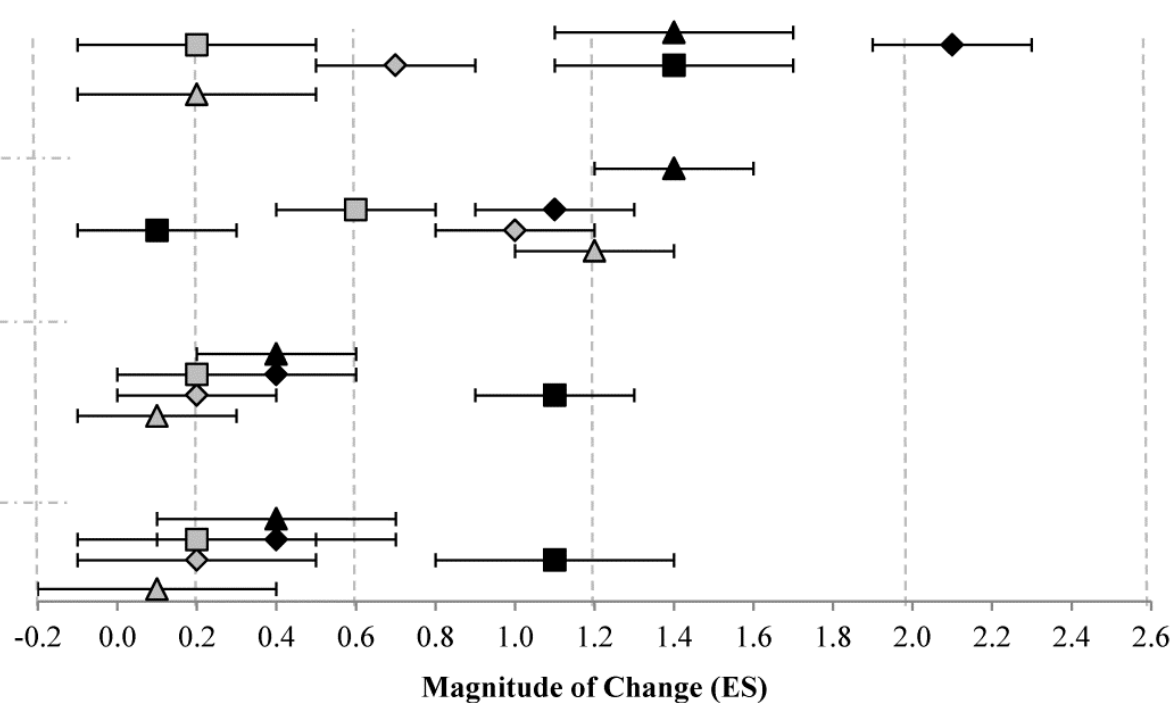


Table 1 - Between-observation variability (CV $\pm 95 \% \mathrm{CL}$ ) and the smallest worthwhile change (\%) required for speed profile measures.

\begin{tabular}{|c|c|c|c|c|c|c|}
\hline & \multicolumn{2}{|c|}{ Overall } & \multicolumn{2}{|c|}{$\mathbf{L P}$} & \multicolumn{2}{|c|}{ HP } \\
\hline & $\mathrm{CV} \%$ & SWC (\%) & CV\% & SWC (\%) & $\mathrm{CV} \%$ & SWC $(\%)$ \\
\hline \multicolumn{7}{|l|}{ Speed profiles } \\
\hline Mean speed $\left(\mathrm{m} \cdot \mathrm{s}^{-1}\right)$ & $1.9 \pm 0.1$ & 2.6 & $2.5 \pm 0.1$ & 1.6 & $1.9 \pm 0.1$ & $1.7^{\text {TuU }}$ \\
\hline Peak speed $\left(\mathrm{m} \cdot \mathrm{s}^{-1}\right)$ & $2.4 \pm 0.2$ & 2.3 & $2.9 \pm 0.1$ & 0.7 & $2.4 \pm 0.1$ & 1.4436 \\
\hline Exercise-intensity ratio (H:L) & $6.8 \pm 0.3$ & 4.4 & $6.8 \pm 0.7$ & 5.1 & $5.8 \pm 0.3$ & ${ }^{3.6} 437$ \\
\hline \multicolumn{7}{|l|}{ High speed activities } \\
\hline Time spent (\%) & $22.4 \pm 0.5$ & 8.9 & $38.8 \pm 0.9$ & 9.7 & $22.4 \pm 0.7$ & $6.4^{400}$ \\
\hline Relative number $\left(\mathrm{n} \min ^{-1}\right)$ & $16.0 \pm 0.1$ & 6.3 & $23.9 \pm 0.2$ & 7.0 & $16.0 \pm 0.1$ & 6.0439 \\
\hline Mean distance (m) & $6.6 \pm 0.9$ & 6.2 & $20.5 \pm 1.6$ & 8.2 & $6.6 \pm 0.9$ & ${ }^{4.5} 440$ \\
\hline Mean duration (s) & $4.5 \pm 0.2$ & 4.7 & $12.5 \pm 0.4$ & 6.0 & $4.5 \pm 0.3$ & 3.6 \\
\hline
\end{tabular}

442 Note: CV - Coefficient of variation; CL - Confidence limits.

443 
445 Table 2 - Descriptive statistics (mean \pm SD) for modified drills in comparison to regular game-simulation drills.

\section{6}

\begin{tabular}{|c|c|c|c|c|}
\hline & \multicolumn{4}{|c|}{ Drills } \\
\hline & 3 vs. 3 & $\begin{array}{l}\text { 30-second } \\
\text { shot-clock }\end{array}$ & $\begin{array}{l}\text { 15-second shot- } \\
\text { clock }\end{array}$ & $\begin{array}{r}\text { Regular } \\
448\end{array}$ \\
\hline Speed profiles & & & & 449 \\
\hline Mean speed $\left(\mathrm{m} \cdot \mathrm{s}^{-1}\right)$ & $1.31 \pm 0.13$ & $1.27 \pm 0.08$ & $1.41 \pm 0.11$ & $1.23 \pm 0.450$ \\
\hline Peak speed $\left(\mathrm{m} \cdot \mathrm{s}^{-1}\right)$ & $3.79 \pm 0.33$ & $3.72 \pm 0.34$ & $3.98 \pm 0.24$ & $\begin{array}{c}3.68 \pm 0.28 \\
451\end{array}$ \\
\hline Exercise-intensity ratio (H:L) & 1:3.1 & $1: 3.7$ & $1: 2.9$ & $1: 3.5$ \\
\hline High speed activities & & & & 452 \\
\hline Time spent (\%) & $4.1 \pm 1.4$ & $2.8 \pm 0.8$ & $3.6 \pm 1.1$ & $2.8 \pm 0.453$ \\
\hline Relative number $\left(\mathrm{n} \min ^{-1}\right)$ & $1.0 \pm 0.4$ & $0.7 \pm 0.2$ & $1.1 \pm 0.4$ & $0.7 \pm 0.454$ \\
\hline Mean distance (m) & $7.1 \pm 1.1$ & $6.6 \pm 1.8$ & $6.0 \pm 2.2$ & $7.1 \pm 1.6455$ \\
\hline Mean duration (s) & $2.2 \pm 0.3$ & $2.0 \pm 0.5$ & $1.6 \pm 0.3$ & $2.1 \pm 0.4$ \\
\hline
\end{tabular}


Table 3 - Descriptive statistics (mean \pm SD) of modified drills for players of different classification compared with regular game-simulation drills.

\begin{tabular}{|c|c|c|c|c|c|c|c|c|}
\hline & \multicolumn{8}{|c|}{ Drills } \\
\hline & \multicolumn{2}{|c|}{3 vs. 3} & \multicolumn{2}{|c|}{ 30-second shot-clock } & \multicolumn{2}{|c|}{ 15-second shot-clock } & \multicolumn{2}{|c|}{ Regular } \\
\hline & LP & HP & LP & HP & LP & HP & LP & HP \\
\hline \multicolumn{9}{|l|}{ Speed profiles } \\
\hline Mean speed $\left(\mathrm{m} \cdot \mathrm{s}^{-1}\right)$ & $1.19 \pm 0.13$ & $1.38 \pm 0.07$ & $1.20 \pm 0.03$ & $1.30 \pm 0.08$ & $1.43 \pm 0.07$ & $1.38 \pm 0.12$ & $1.19 \pm 0.15$ & $1.26 \pm 0.08$ \\
\hline Peak speed $\left(\mathrm{m} \cdot \mathrm{s}^{-1}\right)$ & $3.50 \pm 0.27$ & $3.97 \pm 0.23$ & $3.39 \pm 0.09$ & $3.90 \pm 0.18$ & $3.86 \pm 0.32$ & $4.09 \pm 0.12$ & $3.45 \pm 0.07$ & $3.81 \pm 0.27$ \\
\hline Exercise intensity ratio (H:L) & $1: 2.9$ & $1: 3.1$ & $1: 3.2$ & $1: 4.1$ & $1: 2.2$ & $1: 3.5$ & $1: 3.1$ & $1: 3.8$ \\
\hline \multicolumn{9}{|l|}{ High speed activities } \\
\hline Time spent (\%) & $5.3 \pm 0.8$ & $3.4 \pm 1.1$ & $2.4 \pm 0.5$ & $2.8 \pm 0.8$ & $4.4 \pm 1.2$ & $2.8 \pm 1.1$ & $3.1 \pm 0.5$ & $2.7 \pm 0.8$ \\
\hline Relative number $\left(\mathrm{n} \cdot \mathrm{min}^{-1}\right)$ & $1.2 \pm 0.5$ & $0.8 \pm 0.2$ & $0.8 \pm 0.1$ & $0.7 \pm 0.1$ & $1.3 \pm 0.5$ & $0.9 \pm 0.3$ & $0.8 \pm 0.1$ & $0.6 \pm 0.2$ \\
\hline Mean distance (m) & $6.8 \pm 1.5$ & $7.3 \pm 0.9$ & $4.9 \pm 0.9$ & $7.6 \pm 1.8$ & $6.8 \pm 1.3$ & $6.9 \pm 0.5$ & $6.2 \pm 1.4$ & $7.6 \pm 1.6$ \\
\hline Mean duration (s) & $2.2 \pm 0.4$ & $2.2 \pm 0.3$ & $1.6 \pm 0.3$ & $2.2 \pm 0.5$ & $1.7 \pm 0.4$ & $1.6 \pm 0.2$ & $2.0 \pm 0.4$ & $2.2 \pm 0.3$ \\
\hline
\end{tabular}

461 